OPEN ACCESS

Edited by:

Jiř Fajkus,

Masaryk University, Czech Republic

Reviewed by:

Célia Baroux,

University of Zürich, Switzerland

Martin A. Lysak

Masaryk University, Czech Republic

*Correspondence:

Veit Schubert

schubertv@ipk-gatersleben.de

Specialty section:

This article was submitted to

Plant Cell Biology,

a section of the journal

Frontiers in Plant Science

Received: 02 November 2015

Accepted: 10 January 2016

Published: 15 February 2016

Citation:

Schubert V, Ruban A and Houben A (2016) Chromatin Ring Formation at

Plant Centromeres.

Front. Plant Sci. 7:28.

doi: $10.3389 /$ fpls.2016.00028

\section{Chromatin Ring Formation at Plant Centromeres}

\author{
Veit Schubert ${ }^{1 *}$, Alevtina Ruban ${ }^{1,2}$ and Andreas Houben ${ }^{1}$ \\ ${ }^{1}$ Leibniz Institute of Plant Genetics and Crop Plant Research (IPK) Gatersleben, Stadt Seeland, Germany, ${ }^{2}$ Department of \\ Genetics, Biotechnology, Plant Breeding and Seed Science, Russian State Agrarian University - Moscow Timiryazev \\ Agricultural Academy, Moscow, Russia
}

We observed the formation of chromatin ring structures at centromeres of somatic rye and Arabidopsis chromosomes. To test whether this behavior is present also in other plant species and tissues we analyzed Arabidopsis, rye, wheat, Aegilops and barley centromeres during cell divisions and in interphase nuclei by immunostaining and FISH. Furthermore, structured illumination microscopy (super-resolution) was applied to investigate the ultrastructure of centromere chromatin beyond the classical refraction limit of light. It became obvious, that a ring formation at centromeres may appear during mitosis, meiosis and in interphase nuclei in all species analyzed. However, varying centromere structures, as ring formations or globular organized chromatin fibers, were identified in different tissues of one and the same species. In addition, we found that a chromatin ring formation may also be caused by subtelomeric repeats in barley. Thus, we conclude that the formation of chromatin rings may appear in different plant species and tissues, but that it is not specific for centromere function. Based on our findings we established a model describing the ultrastructure of plant centromeres and discuss it in comparison to previous models proposed for animals and plants.

Keywords: CENH3, centromere organization, interphase nucleus, meiosis, mitosis, repetitive DNA, superresolution microscopy

\section{INTRODUCTION}

Centromeres of eukaryotic chromosomes are regions where spindle fibers attach to perform chromatid or homolog separation during mitosis and meiosis. Structurally, different types of centromeres exist (Cuacos et al., 2015). Commonly, they represent a distinct single primary constriction (monocentric chromosomes). But they may also be undiscernible (no primary constriction at very small chromosomes), as e.g., described in Giardia intestinalis Kofoid and Christiansen 1915 (Tůmová et al., 2015). Primary constrictions can be elongated to several microns (polycentric chromosomes) as found e.g., in wallaby hybrids (Metcalfe et al., 2007), Lathyrus and pea (Neumann et al., 2012, 2016), or form a groove along both sister chromatids of holocentric chromosomes, e.g., of the wood rush Luzula elegans LOWE (Heckmann et al., 2011; Wanner et al., 2015).

Centromeres are not conserved at the DNA sequence level and evolutionary long-established centromeres are frequently formed on long arrays of satellite repeat DNAs and/or transposable elements (Henikoff et al., 2001; Jiang et al., 2003; Plohl et al., 2014). In most eukaryotes the histone 
variant $\mathrm{CENH} 3$ serves as a marker for centromeric chromatin and assembles in many species on such specific families of repetitive DNA sequences (Houben and Schubert, 2003; Marques et al., 2015).

Animal centromeres form a trilaminar chromatin-protein complex composed of centromere chromatin and kinetochore proteins (Blower et al., 2002; Sullivan and Karpen, 2004; Ribeiro et al., 2010; Screpanti et al., 2011). Contrary, for meiotic plant centromeres a "ball in a cup" kinetochore configuration was postulated (Bajer and Mole-Bajer, 1972; Dawe et al., 2005). Ultrastructural studies at somatic metaphase centromeres of plants showed that CENH3-containing chromatin forms "curved pad" structures at the surface and sub-surface periphery of the primary constriction where spindle fibers attach (Wanner et al., 2015).

Previously, we identified the formation of ring-like structures at somatic metaphase chromosomes of rye by immunostaining with CENH3-specific antibodies (Banaei-Moghaddam et al., 2012) and in Arabidopsis thaliana (L.) Heynh. in interphase nuclei using a centromere-specific repetitive DNA sequence as FISH probe (Schubert et al., 2012). In this study we applied Structured Illumination Microscopy (SIM) which allows the identification of structures beyond the diffraction limit of light at a lateral resolution of $\sim 120 \mathrm{~nm}$ and an axial resolution of $\sim 250 \mathrm{~nm}$ (Schermelleh et al., 2010), to clarify whether centromeric chromatin ring formation is a common feature at plant monocentromeres. Therefore, we investigated Arabidopsis and cereal centromeres during cell division and interphase in different tissues.

\section{MATERIALS AND METHODS}

\section{Plant Material}

The following species were analyzed: Arabidopsis thaliana (L.) Heynh. $(2 \mathrm{n}=10)$, Secale cereale L. (rye, $2 \mathrm{n}=14)$, Triticum aestivum L. (wheat, $2 \mathrm{n}=42$ ), Aegilops speltoides ssp. aucheri (Boiss.) Chennav. ( $2 \mathrm{n}=14+$ supernumerary B chromosomes) and Hordeum vulgare L. (barley, $2 \mathrm{n}=14$ ).

\section{Slide Preparation, Immunostaining, and FISH}

Flower buds and/or root tips of wheat, rye and barley were fixed for $45 \mathrm{~min}$ in ice-cold $4 \%(\mathrm{w} / \mathrm{v})$ paraformaldehyde in 1xMTSB buffer (50 mM PIPES, $5 \mathrm{mM} \mathrm{MgSO} 4$, and $5 \mathrm{mM}$ EGTA, pH 7.2). After washing in $1 \mathrm{xMTSB}$, chromosome spreads were prepared by squashing. Young Ae. speltoides spikes were pretreated in icecold water for $24 \mathrm{~h}$ and then fixed in ethanol:acetic acid (3:1) for at least 4 days. Afterwards, the spikes were stained with acetocarmine and chromosomes were prepared by squashing in $45 \%$ acetic acid.

Tissue sections of Ae. speltoides were prepared according to Steedman (1957) and Braszewska-Zalewska et al. (2013). Briefly, developing seeds were excised from spikelets and fixed in freshly prepared 3\% (w/v) paraformaldehyde with $0.05 \%$ Triton X100 in $1 \times$ PBS buffer on ice for $5 \mathrm{~h}$. Then, dehydration was performed in an ethanol series (ethanol/1 $\times$ PBS buffer) from 30 to $96 \%$ for
$30 \mathrm{~min}$ in each at room temperature and in $96 \%$ for $30 \mathrm{~min}$ at $37^{\circ} \mathrm{C}$. Afterwards, the tissues were infiltrated with PEG1500-wax and embedded in a small casting mold (1-3 seeds per block). The blocks were cut into $10 \mu \mathrm{m}$ slices using a Leica microtome (RM2265; knifes 35N from Feather company). The slices were transferred onto poly-L-lysine-coated slides using forceps and a brush and were stretched by adding a drop of $1 \mu l$ water over each slice. The rest of PEG-wax was removed from the dry slides by washing them in $90 \%$ ethanol.

Differentiated 2-16C leaf nuclei of A. thaliana were isolated and flow sorted according to their DNA content from differentiated rosette leaves after formaldehyde fixation using a FACS Aria (BD Biosciences) as described (Pecinka et al., 2004).

To evaluate the substructure of $\mathrm{CENH} 3$ containing chromatin, immunostaining was performed according to Jasencakova et al. (2000). CENH3 was detected with rabbit anti-grass CENH3 primary antibodies (Sanei et al., 2011) and goat anti-rabbit rhodamine (1:300; Jackson Immuno Research Laboratories) or goat anti-rabbit Alexa488 secondary antibodies (1:200; Molecular Probes). Spindle fibers were labeled with monoclonal mouse anti $\alpha$-tubulin (1:200; clone DM 1A, Sigma) and anti-mouse Alexa488 (1:400; Molecular Probes) antibodies.

For FISH the 180-bp centromeric repeat sequence pAL of A. thaliana (Martinez-Zapater et al., 1986) was generated by PCR as described (Kawabe and Nasuda, 2005). The centromeric retrotransposon CRW2 of wheat was generated by PCR as described by Li et al. (2013). These probes as well as the subtelomeric repeat HvT01 (Schubert et al., 1998) and BAC7 containing centromere-specific repeats of barley (Hudakova et al., 2001; Houben et al., 2007) were directly labeled by nick translation with TexasRed-dUTP, Alexa488-dUTP and Cy3dUTP according to Ward (2002). FISH was performed according to Schubert et al. (2001).

For the colocalization of CENH3 immunosignals with centromeric FISH signals, immunostaining was performed first. The slides were treated with $10 \mathrm{mM}$ citrate buffer $(\mathrm{pH}$ 6) in a microwave at 800 Watt for $60 \mathrm{~s}$ according to Chelysheva et al. (2010). Then the primary antibodies were applied and immunostaining was performed as described (Jasencakova et al., 2000). Prior FISH the slides were treated with ethanol:acetic acid (3:1) fixative for $10 \mathrm{~min}$ and freshly prepared $4 \%$ formaldehyde in $1 \times$ PBS for $10 \mathrm{~min}$, followed by three times washing for $5 \mathrm{~min}$ in $1 \times$ PBS. These steps are important to stabilize the immunosignals during the following FISH procedure, which was performed as described (Ma et al., 2010). Nuclei and chromosomes were counterstained with DAPI $(1 \mu \mathrm{g} / \mathrm{ml})$ in Vectashield (Vector Laboratories).

\section{Super-Resolution Microscopy}

To analyse the substructure of chromatin beyond the classical Abbe/Raleigh limit (super-resolution) spatial Structured Illumination Microscopy (3D-SIM) was applied using a C-Apo $63 \times / 1.2$ W Korr objective of an Elyra PS.1 microscope system and the software ZEN (Carl Zeiss $\mathrm{GmbH}$ ). Images were captured using 405, 488, and $561 \mathrm{~nm}$ laser lines for excitation (42, 34, and $28 \mu \mathrm{m}$ grids for 561,488 , and $405 \mathrm{~nm}$ excitations; 5 rotations) and the appropriate emission filters. 3D-SIM stacks with a step 
size of $110 \mathrm{~nm}$ were acquired consecutively for each fluorophore starting with the highest wavelength dye to minimize bleaching. SIM image stacks were used to produce $3 \mathrm{D}$ movies by the Imaris 8.0 (Bitplane) and ZEN 2012 software.

\section{RESULTS}

We applied centromere- and subtelomere-specific DNA repeats and antibodies against $\mathrm{CENH} 3$ to investigate the ultrastructure of specifically labeled chromatin and to check whether the chromatin ring formation is a specific feature of centromeres in monocot plants and the eudicot species A. thaliana.

\section{Centromere Chromatin Ring Formation Appears in Arabidopsis Nuclei of Different Endopolyploidy}

To test whether there is a varying centromeric chromatin ring formation in interphase nuclei of different endopolyploidy, flow sorted $2-16 \mathrm{C}$ differentiated leaf nuclei of $A$. thaliana were analyzed using the centromere-specific DNA repeat pAL as FISH probe. In addition to globular structures, also ring and halfring structures were identified at all ploidy levels. By SIM it became obvious that these centromere structures are composed of a network of chromatin loops (Figure 1). Ring and halfring formation appears around chromocenters and especially after centromere association, meaning that centromeres tend to fuse in interphase nuclei. In 16C nuclei due to decreased cohesion aligned centromeric chromatid regions start to separate (Schubert et al., 2006). Then, more than 10 centromere signals (corresponding to the chromosome number in A. thaliana) may appear. These centromere regions keep the globular substructure, but no further ring formation was observed (Figure 1).

\section{Centromere Chromatin Ring Formation is Present at Rye and Wheat Chromosomes during Mitosis and Meiosis}

To investigate the ultrastructure of active centromeres during cell division, rye centromeres were labeled with CENH3-specific antibodies and analyzed by SIM. During mitosis and meiosis as well as in interphase nuclei of roots and anthers CENH3 containing chromatin domains form ring-like structures measuring $\sim 0.5-1.0 \mu \mathrm{m} \quad$ (Figure 2; Supplementary Movies 1-12). When these rings belonging to single or paired homologs, comprising the centromeres of either two or four sister chromatids, associate or align (e.g., in interphase and prophase I, respectively), they fuse and form bigger rings. During late somatic and meiotic prophase these rings split again and compose smaller ones clearly visible at somatic metaphase chromosomes and at bivalents. Already in metaphase I some of the rings of the sister centromeres split again and become then distantly separated in interkinesis. Similarly, the formation of ring-like structures by CENH3 containing nucleosomes was observed during meiosis of hexaploid wheat (Figure 3).

\section{Centromere Chromatin Ring Formation Varies Between Aegilops Tissues}

Anti-CENH3 and the centromere-specific repeat CRW2 (Liu et al., 2008) were used to analyse the centromere substructures of Ae. speltoides. In most embryonic interphase nuclei the CENH3-labeling shows clearly a ring formation by looped chromatin fibers (Figures $\mathbf{4 A}_{\mathbf{1}, \mathbf{2}}$ ) but not at metaphase centromeres (Figure $\mathbf{4 A}_{\mathbf{3}}$ ). Irrespective of the presence of supernumerary $\mathrm{B}$ chromosomes all chromosomes showed the same centromere chromatin substructure, although we could not identify the $\mathrm{Bs}$ based on $\mathrm{B}$-specific probes (Figure $\mathbf{4 A}_{\mathbf{3}}$; $2 n=14+2 \mathrm{~B})$.

In spike meristems both CENH3 and CRW2 form spherical reticulate substructures during the somatic cell cycle which intermingle among each other. But no ring formation was observed (Figures $\mathbf{4 B}_{\mathbf{1 - 4}}$ ). The differently labeled, but with $\mathrm{CENH} 3$ chromatin intermingled CRW2 repeat fibers indicate that most of CRW2 is not associated with CENH3 (Figures $\mathbf{4 A}_{\mathbf{1}}, \mathbf{B}_{\mathbf{1 - 4}}$ ). The supernumerary chromosomes in pro-metaphase (Figure $4 \mathrm{~B}_{2} ; 2 \mathrm{n}=14+1 \mathrm{~B}$ ) and metaphase (Figure $\mathbf{4 B}_{3} ; 2 \mathrm{n}=14+2 \mathrm{~B}$ ) obviously do not show a deviating centromere structure.

Compared to embryo nuclei, endosperm nuclei exhibit a more compact $\mathrm{CENH} 3$ chromatin organization. Thus, a ring formation is less often visible (Figure 4C).

We conclude that the centromere chromatin organization may differ between tissues of individual Ae. speltoides plants.

\section{Chromatin Ring Formation Appears also Outside of Barley Centromeres}

CENH3 antibodies and the centromere-specific repeat containing BAC7 probe of barley (Hudakova et al., 2001; Houben et al., 2007) were applied to label the centromere substructures of barley. For comparison, also the subtelomeric repeat HvT01 (Schubert et al., 1998) was applied as a FISH probe.

In interphase nuclei both $\mathrm{CENH} 3$-positive chromatin and centromeric repeats may establish ring structures (Figures $\mathbf{5} \mathbf{A}_{\mathbf{1 - 4}}$ ). CENH3 chromatin is embedded in centromeric repeats as a condensed globular structure or CENH3-ring-incentromere repeat-ring configurations may appear. During the somatic cell cycle the centromeric repeats compose reticulate substructure. This is true also for the subtelomeric repeat HvT01, but it may also compose ring chromatin structures (Figures $\mathbf{5 B}_{\mathbf{1 - 3}}$ ). The observation that in identical nuclei differently shaped chromatin substructures occur, excludes that preparation artifacts inducing the ring structure formation arose.

In short, we conclude that the formation of chromatin rings is not a specific feature of centromeres.

\section{DISCUSSION}

Applying super-resolution microscopy we investigated the ultrastructure of centromere chromatin. We found that in different monocot plants and the eudicot species A. thaliana centromeric chromatin fibers may establish globular and/or 

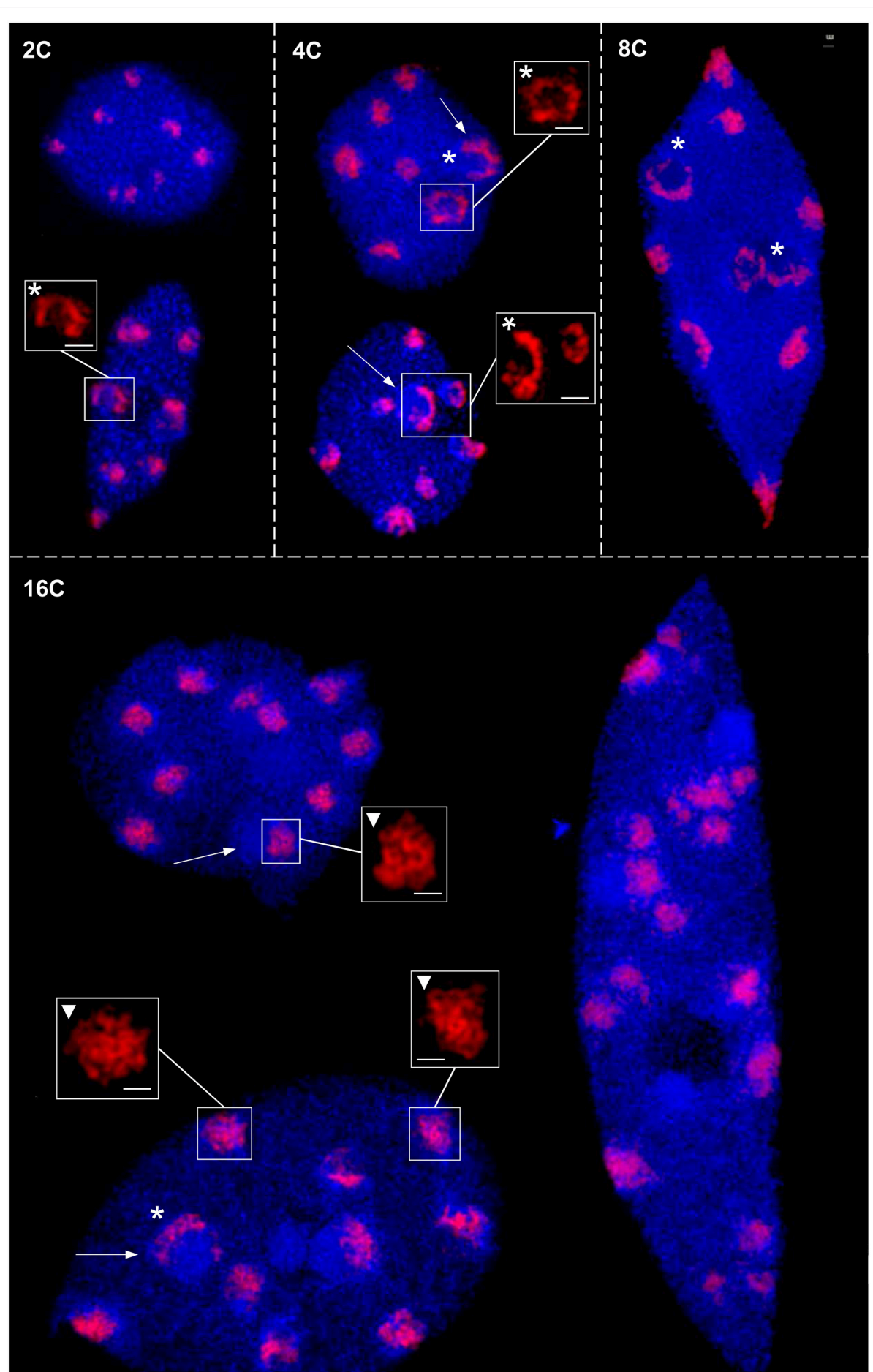

PAL-repeat

FIGURE 1 | Centromeric chromatin (pAL) ring formation in differentiated 2-16C leaf nuclei of $\boldsymbol{A}$. thaliana. In addition to globular reticulate chromatin structures (triangles), ring and half-ring formation (asterisks) appears at all ploidy levels, especially around chromocenters (arrows). In 16C nuclei due to loss of cohesion centromeres may become separated but they keep their globular structure as demonstrated by the 17 signals in the right nucleus. Bar size in inset $=0.5 \mu \mathrm{m}$.

pad-like structures. But also ring structures may appear with varying peculiarities in cycling and differentiated cells depending on the tissue analyzed.
In addition, we demonstrated, although less pronounced, that also chromatin containing subtelomeric repeats may be organized in a ring-like manner. This suggests that 

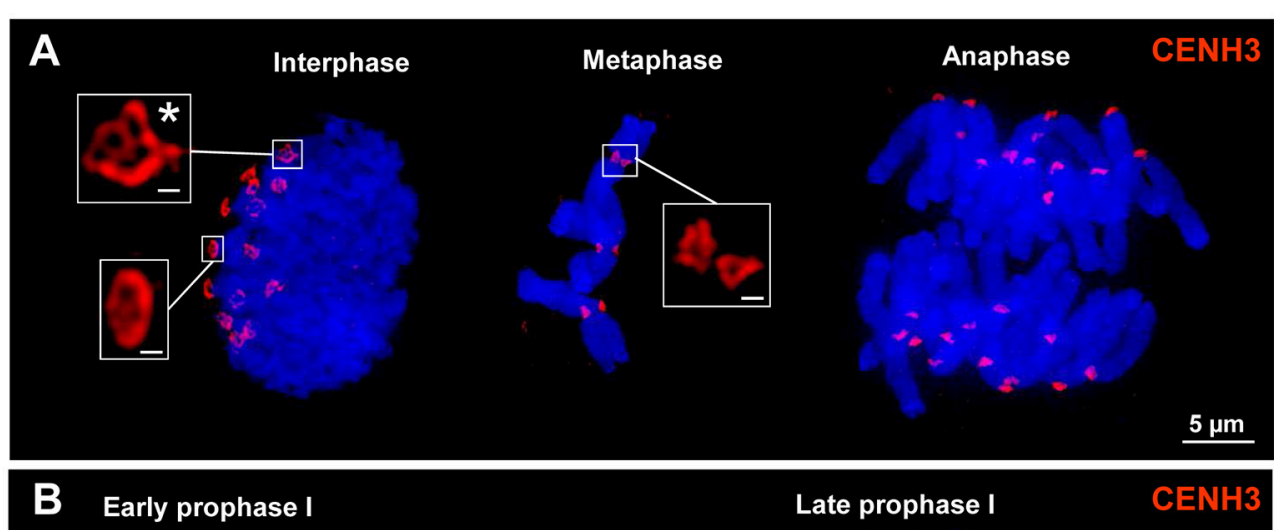
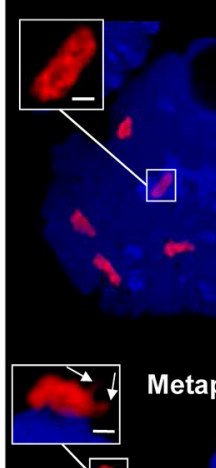

Metaphase
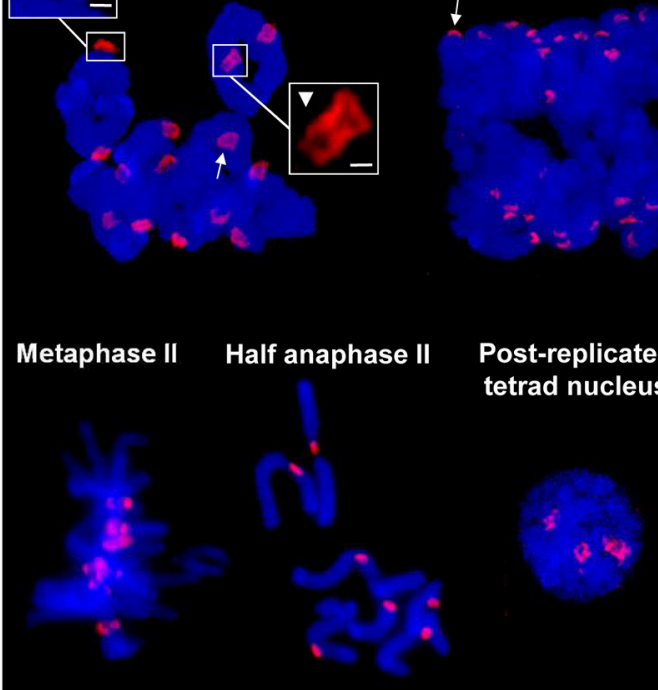

Anaphase I
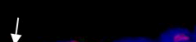

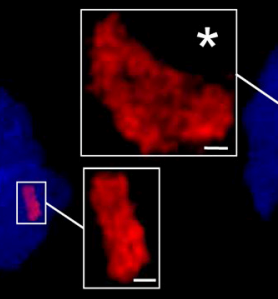

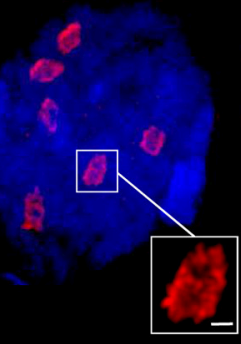

Interkinesis
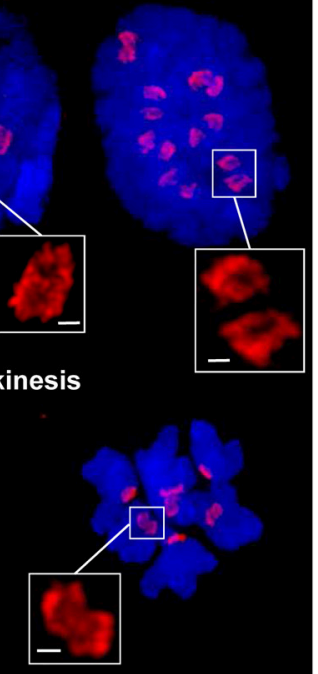

\section{Two tapetum nuclei Somatic interphase}

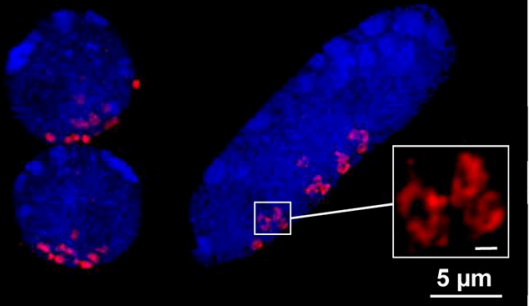

FIGURE 2 | CENH3 chromatin ring formation at centromeres of rye during mitosis, meiosis and in interphase nuclei. (A) The 14 CENH3 labeled centromeres visible as rings in interphase become separated at somatic metaphase which segregate at their chromatids during anaphase. The main ring structures may be formed by several subrings (asterisk). (B) CENH3 structures during meiosis in pollen mother cells and somatic anther cells. After centromere alignment in early prophase I homologous centromeres coalesce and form ring structures which are split at the end of prophase I. The single rings composed by the two sister centromers present in metaphase I start to separate again (triangle) and in interkinesis clearly two rings are visible at each centromere. They are required to separate the sister chromatids in anaphase II. In prophase I centromeres may also associate (asterisk). At metaphase I and anaphase I bivalents the CENH3 chromatin ring structures are characterized by a cap/crown-like shape comprising extensions where spindle fibers may attach (arrows; see also Supplementary Movies 7-12). Somatic interphase nuclei (two of them in tapetum cells, and single ones in other cells) from anthere tissues show Rabl orientation (Rabl, 1885). Also in these nuclei CENH3 structures may coalesce and form ring structures. Bar size in inset $=0.5 \mu \mathrm{m}$.

repetitive DNA sequences tend to associate as found for A. thaliana centromeric (Schubert et al., 2012, 2013) and transgenic repeats along chromosome arms (Pecinka et al., 2005; Jovtchev et al., 2008, 2011; Watanabe et al.,
2009). Self-organization and fractal globule formation of chromatin may be models to explain the arrangement of such chromatin segments and its dynamics (Misteli, 2007, 2009; McNally and Mazza, 2010). The observation that non-cycling 


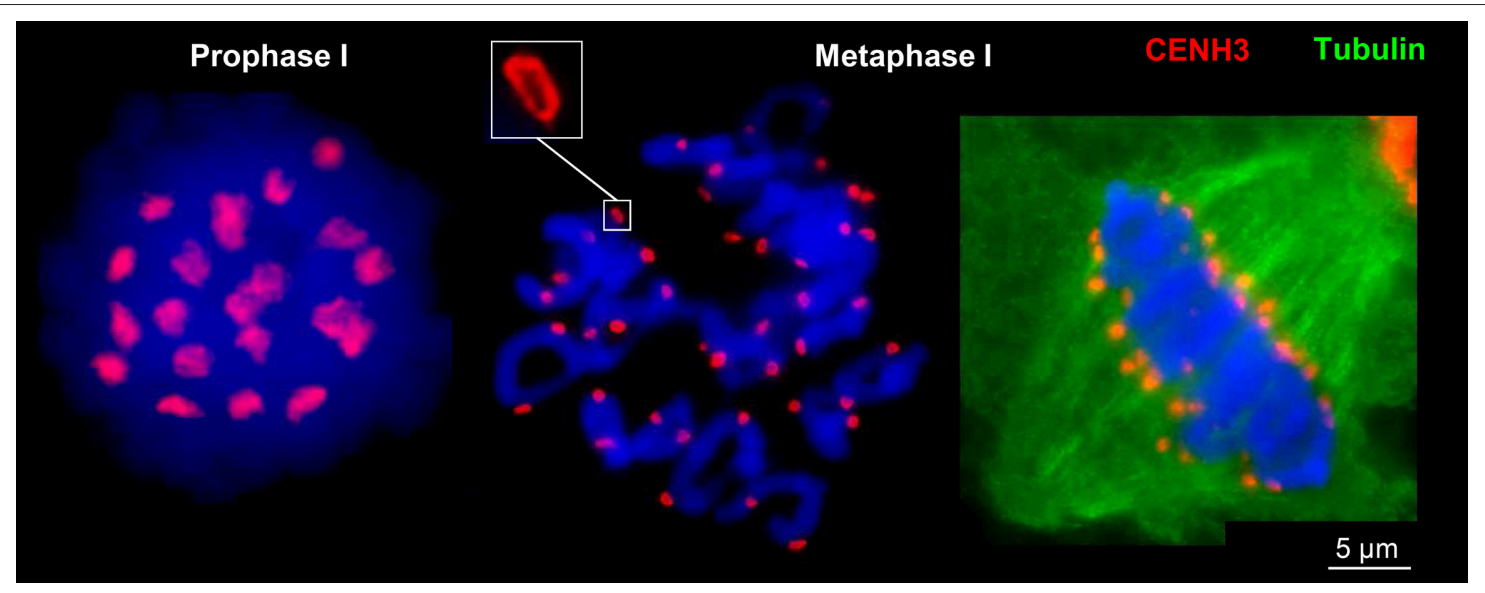

FIGURE 3 | CENH3-positive centromere structures during meiosis of hexaploid wheat. In prophase I the four centromeres of paired sister chromatids of both homologs are fused and form ring-like structures which become separated in metaphase I where only two sister chromatids are fused and spindle fibers attach (right).

differentiated endoreduplicated A. thaliana nuclei also establish chromatin ring structures at centromeres indicates that this structure is possibly not absolutely required for spindle fiber attachment.

Thus, although there is increasing evidence that an interplay between higher-order chromatin organization and function of nuclei exists (Woodcock and Ghosh, 2010), it remains unclear whether centromeric chromatin rings are essential for kinetochore formation and the attachment of spindle fibers. At least the findings of Bailey et al. (2013) demonstrate that a secondary centromere structure may be important. They showed in human HeLa cells that epigenetic modifications of the N-terminal tail of $\mathrm{CENH3}$ alter the physical properties of chromatin fibers in such a way that the local centromere chromatin organization may be influenced.

The monocentromeres of barley (Ishii et al., 2015), but also the polycentromeres of Pisum and Lathyrus (Neumann et al., 2016) have two different CENH3 variants. Both establish globular structures formed by intermingling chromatin fibers, but no centromeric ring formation was observed at somatic metaphase chromosomes. This corresponds to the finding here that the centromeric BAC7 repeats of barley show chromatin fibers only spherically organized at somatic metaphase. The occurrence of pad-like structures at monocentric somatic plant centromeres has also been confirmed by high resolution scanning electron microscopy studies. It became obvious that spindle fibers attach mainly to the pericentromeric flanks of the primary constriction, probably to transfer at least a part of the pulling forces from the microtubules to the more stable chromosome arms to prevent chromosome breakage (Wanner et al., 2015) (Figure 6). This may be one reason why naturally occurring telocentric chromosomes are seldom (Darlington, 1939). However, the telosomes of artificially selected wheat lines containing about half of the CENH3 chromatin amount compared to complete chromosomes are relatively stable (Koo et al., 2015). The prove of Zhang and
Dawe (2012) that total centromere size and chromosome size are positively correlated in grass species supports the idea that an adequate amount of $\mathrm{CENH} 3$ chromatin is required to maintain the chromosome stability during cell divisions.

Interestingly, scanning electron microscopy studies of meiotic Tradescantia reflexa Raf. chromosomes revealed that the centromeres of metaphase I and anaphase I bivalents exhibit a ring-like structure measuring $0.5-0.7 \mu \mathrm{m}$ in diameter. This structure is localized in a crown-like manner polewards at the bivalents and contains small extensions (Inaga et al., 2000). These observations correspond clearly to shape and size of the centromere ultrastructure we identified by SIM during the meiosis of rye.

Obviously, plant monocentromeres establish a centromere structure deviating from the trilaminar structure of mammals and insects. The ball-in-a-cup organization postulated by Bajer and Mole-Bajer (1972) and adapted by Dawe et al. (2005) for meiotic metaphase I chromosomes of maize may be related to our findings of globular, pad- and/or ring-like CENH3 chromatin structures in such a way that, as we proved here, the CENH3-positive chromatin is mostly centrally embedded in more extended pericentromeric heterochromatin (Figure 6).

In contrast to the B chromosome of rye (Banaei-Moghaddam et al., 2012) we did not find differences regarding the distribution of centromeric repeats between $\mathrm{A}$ and $\mathrm{B}$ chromosomes of Ae. speltoides. However, as we included in our study only one centromeric repeat it remains open whether other centromeric wheat repeats differ between $\mathrm{A}$ and $\mathrm{B}$ chromosomes in $\mathrm{Ae}$. speltoides.

Altogether, we conclude, that centromere ring formation may vary between tissues of one and the same species, between closely related species, but may also be present in more distantly related species. This ring formation may be a matter of repeat self-organization and involved, but not specific for centromere function. 


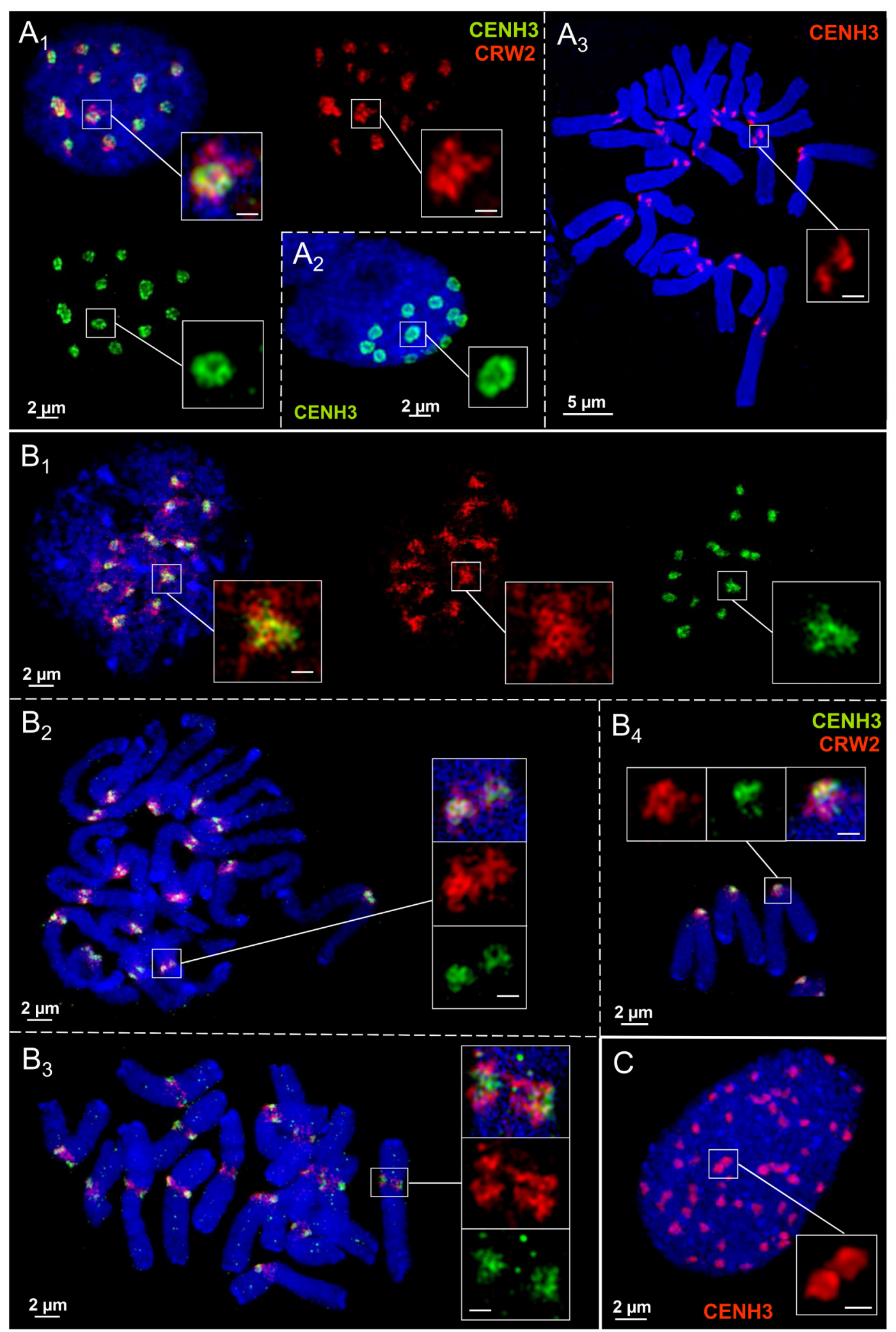

FIGURE 4 | Centromere chromatin substructures in embryo (A), spike (B), and endosperm (C) tissues of Ae. speltoides. CENH3 chromatin ring formation appears pronounced in embryo interphase nuclei $\left(\mathbf{A}_{\mathbf{1}-\mathbf{2}}\right)$, but not at metaphase chromosomes $\left(\mathbf{A}_{\mathbf{3}}\right)$. The centromeric CRW2 repeat shows mainly a globular organization formed by chromatin fibers present also for CENH3 chromatin in spike tissue during the cell cycle $\left(\mathbf{B}_{1-4}\right)$. Due to a high degree of chromatin condensation the CENH3 chromatin ring formation is less clearly visible in endosperm nuclei (C). Bar size in inset $=0.5 \mu \mathrm{m}$. 

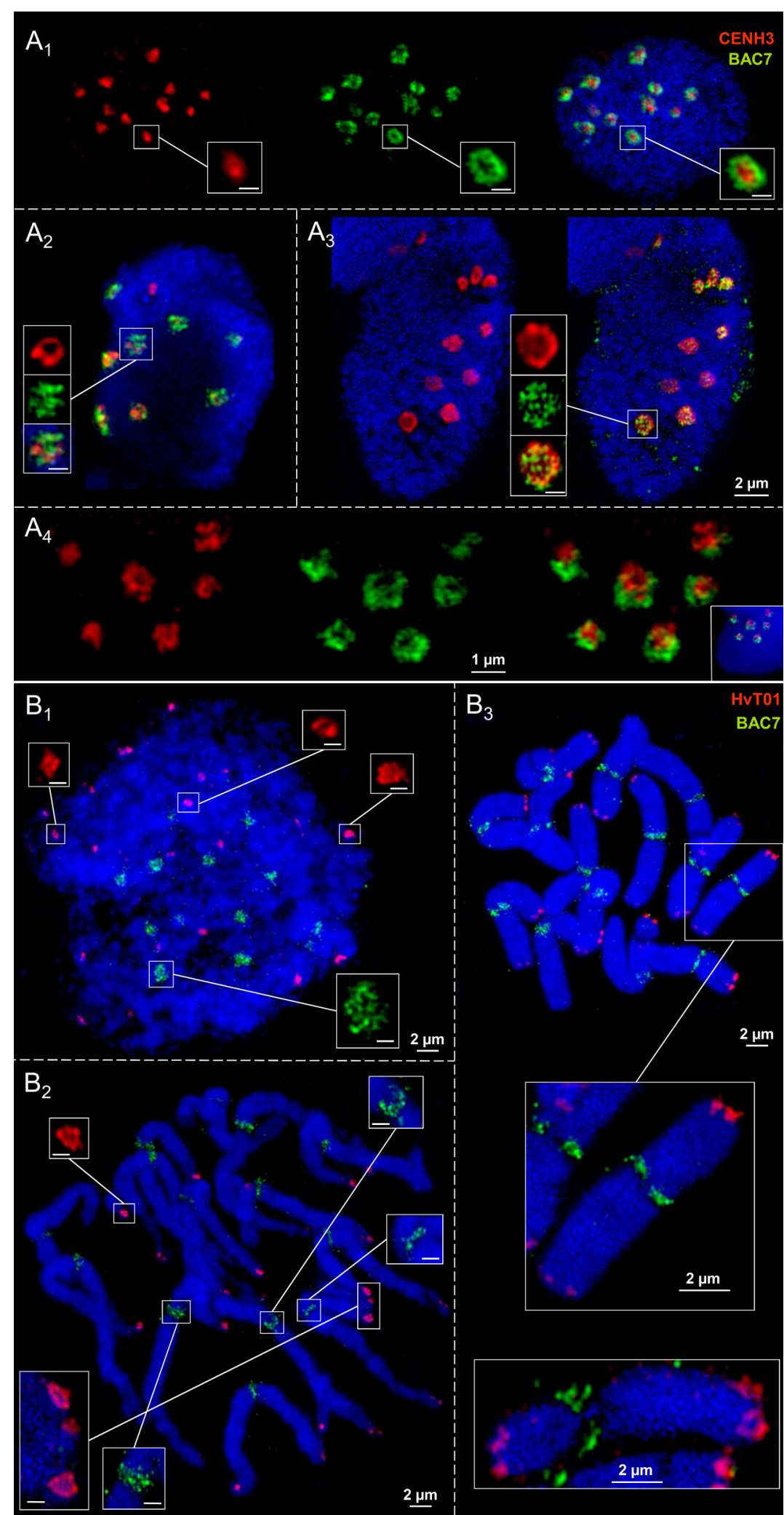

FIGURE 5 | Ultrastructural organization of centromeres and subtelomeric regions at metaphase chromosomes and in interphase nuclei of barley. Anti-CENH3 and centromere-specific BAC7-repeats were used as centromeric probes. Repeat HvT01 detects the subtelomeric regions. In interphase nuclei CENH3 chromatin may compose highly condensed globular structures embedded in ring chromatin containing centromeric repeats $\left(\mathbf{A}_{\mathbf{1}}\right)$. Alternatively, $\mathrm{CENH} 3$ chromatin may (Continued) 
FIGURE 5 | Continued

show ring structures and the centromere repeats (BAC7) may be organized by chromatin fibers in a less condensed globular manner $\left(\mathbf{A}_{\mathbf{2}-3}\right)$. In addition, both $\mathrm{CENH3}$ and centromeric BAC7 repeats may form rings $\left(\mathbf{A}_{\mathbf{4}}\right) .\left(\mathbf{B}_{1-3}\right)$ While the centromeric repeats (BAC7) establish reticulate chromatin substructures at centromeres during the somatic cell cycle at centromeres, subtelomeric repeats (HvT01) may form ring-like structures at the subtelomeres in interphase $\left(\mathbf{B}_{\mathbf{1}}\right)$, prometaphase $\left(\mathbf{B}_{\mathbf{2}}\right)$, and metaphase $\left(\mathbf{B}_{3}\right)$. Bar size in insets $=0.5 \mu \mathrm{m}$.

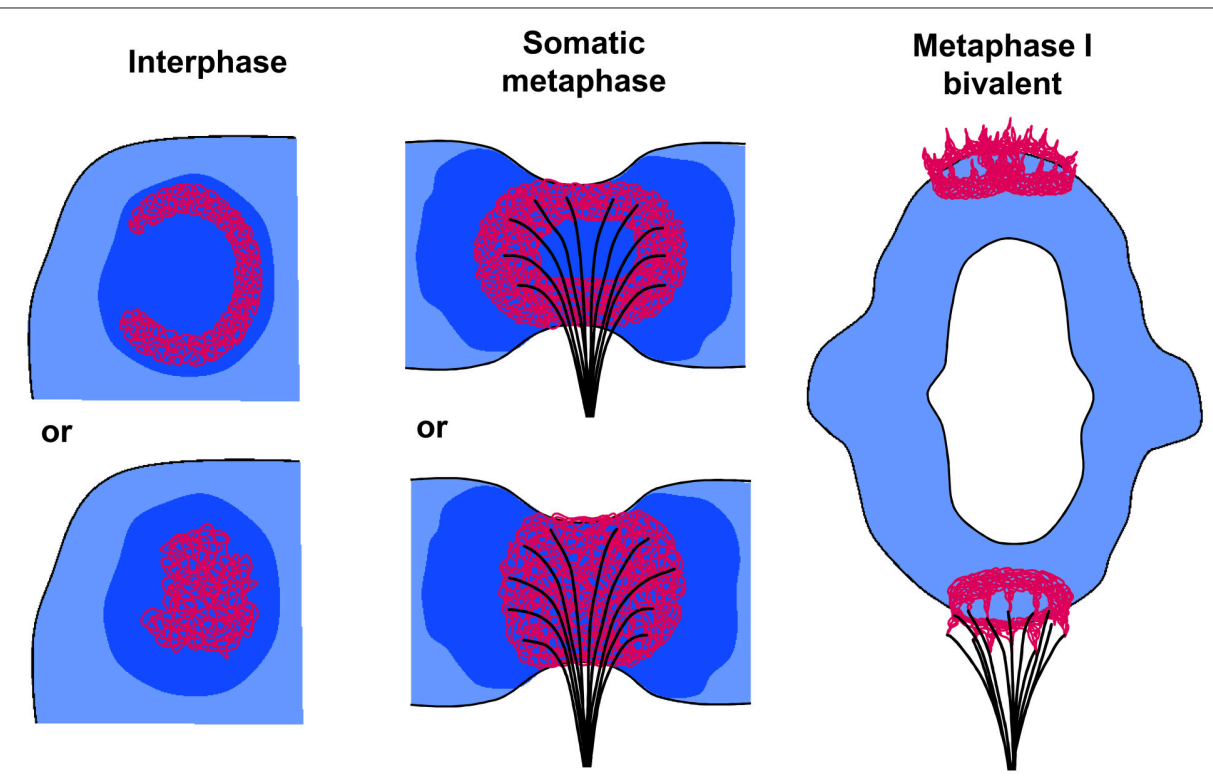

FIGURE 6 | Models of plant centromere chromatin organization at interphase, somatic metaphase, and metaphase I chromosomes. In interphase CENH3-containing chromatin (red) may form ring-like (top) or globular (bottom) structures embedded in CENH3-negative heterochromatin (dark blue). At somatic metaphase chromosomes (side view of a single chromatid) the CENH3 chromatin forms rings (top) or globular/pad-like (bottom) structures where spindle fibers attach. The CENH3 chromatin is surrounded by pericentromeric heterochromatin (dark blue). The spindle fibers attach mainly to the pericentromeric flanks of the primary constriction to transfer pulling forces from the microtubules to the more stable chromosome arms (see also Wanner et al., 2015). At metaphase I bivalents, single fused CENH3 chromatin rings composed by the two sister centromers show a crown- like shape (bottom homolog) due to the pulling forces of the microtubules. They become separated again (top homolog) during the transition to anaphase I.

\section{AUTHOR CONTRIBUTIONS}

VS conceived the study and designed the experiments. VS and AR performed the experiments. VS and AH wrote the manuscript. All authors read and approved the final manuscript.

\section{ACKNOWLEDGMENTS}

We thank Jörg Fuchs for flow sorting of nuclei, Andrea Kunze, Martina Kühne, and Joachim Bruder for excellent assistance, and Karin Lipfert for artwork.

\section{SUPPLEMENTARY MATERIAL}

The Supplementary Material for this article can be found online at: http://journal.frontiersin.org/article/10.3389/fpls.2016. 00028

Supplementary Movie 1 | Somatic oval rye interphase nucleus showing Rabl orientation and ring formation of CENH3 chromatin at centromeres.

Supplementary Movie 2 | Somatic elongated rye interphase nucleus showing Rabl orientation and ring-like formation of CENH3 chromatin at centromeres.
Supplementary Movie 3 | Early prophase I of rye showing seven mostly aligned pairs of homologous centromeres.

Supplementary Movie 4 | Mid prophase I of rye where two pairs of homologous centromeres form already rings.

Supplementary Movie 5 | Late prophase I of rye where all seven paired homologous centromeres form ring-like structures.

Supplementary Movie 6 | Late prophase I of rye where one pair of homologous centromeres (center) split into two rings.

Supplementary Movie 7 | Metaphase I of rye with CENH3 chromatin ring formation at the seven bivalents.

Supplementary Movie 8 | Metaphase I of rye with CENH3 chromatin ring formation at the seven bivalents. One pair of homologous centromeres (enlarged) split already into two rings required for spindle fiber attachment in meiosis II.

Supplementary Movie 9 | Metaphase I of rye with a mainly reticulate pad-like CENH3 chromatin arrangement at the seven bivalents.

Supplementary Movie 10 | Three rye bivalents with CENH3 chromatin showing a cap/crown-like shape induced by spindle fiber tension.

Supplementary Movie 11 | Rye bivalent with CENH3 chromatin showing a cap/crown-like and reticulate organization.

Supplementary Movie 12 | Rye bivalent with a dense CENH3 chromatin organization. 


\section{REFERENCES}

Bailey, A. O., Panchenko, T., Sathyan, K. M., Petkowski, J. J., Pai, P. J., Bai, D. L., et al. (2013). Posttranslational modification of CENP-A influences the conformation of centromeric chromatin. Proc. Natl. Acad. Sci. U.S.A. 110, 11827-11832. doi: 10.1073/pnas.1300325110

Bajer, A. S., and Mole-Bajer, J. (1972). Spindle Dynamics and Chromosome Movements. New York, NY: Academic Press.

Banaei-Moghaddam, A. M., Schubert, V., Kumke, K., Weibeta, O., Klemme, S., Nagaki, K., et al. (2012). Nondisjunction in favor of a chromosome: the mechanism of rye B chromosome drive during pollen mitosis. Plant Cell 24, 4124-4134. doi: 10.1105/tpc.112.105270

Blower, M. D., Sullivan, B. A., and Karpen, G. H. (2002). Conserved organization of centromeric chromatin in flies and humans. Dev. Cell 2, 319-330. doi: 10.1016/S1534-5807(02)00135-1

Braszewska-Zalewska, A. J., Wolny, E. A., Smialek, L., and Hasterok, R. (2013). Tissue-specific epigenetic modifications in root apical meristem cells of Hordeum vulgare. PLoS ONE 8:e69204. doi: 10.1371/journal.pone.0069204

Chelysheva, L., Grandont, L., Vrielynck, N., le Guin, S., Mercier, R., and Grelon, M. (2010). An easy protocol for studying chromatin and recombination protein dynamics during Arabidopsis thaliana meiosis: immunodetection of cohesins, histones and MLH1. Cytogenet. Genome Res. 129, 143-153. doi: $10.1159 / 000314096$

Cuacos, M., Franklin, F., and Heckmann, S. (2015). Atypical centromeres in plants - what they can tell us. Front. Plant Sci. 6:913. doi: 10.3389/fpls.2015.00913

Darlington, C. D. (1939). Misdivision and the genetics of the centromere. J. Genet. 37, 343-365. doi: 10.1007/BF02982733

Dawe, R. K., Richardson, E. A., and Zhang, X. (2005). The simple ultrastructure of the maize kinetochore fits a two-domain model. Cytogenet. Genome Res. 109, 128-133. doi: 10.1159/000082391

Heckmann, S., Schroeder-Reiter, E., Kumke, K., Ma, L., Nagaki, K., Murata, M., et al. (2011). Holocentric chromosomes of Luzula elegans are characterized by a longitudinal centromere groove, chromosome bending, and a terminal nucleolus organizer region. Cytogenet. Genome Res. 134, 220-228. doi: $10.1159 / 000327713$

Henikoff, S., Ahmad, K., and Malik, H. S. (2001). The centromere paradox: stable inheritance with rapidly evolving DNA. Science 293, 1098-1102. doi: $10.1126 /$ science. 1062939

Houben, A., Schroeder-Reiter, E., Nagaki, K., Nasuda, S., Wanner, G., Murata, M., et al. (2007). CENH3 interacts with the centromeric retrotransposon cereba and GC-rich satellites and locates to centromeric substructures in barley. Chromosoma 116, 275-283. doi: 10.1007/s00412-007-0102-z

Houben, A., and Schubert, I. (2003). DNA and proteins of plant centromeres. Curr. Opin. Plant Biol. 6, 554-560. doi: 10.1016/j.pbi.2003.09.007

Hudakova, S., Michalek, W., Presting, G. G., ten Hoopen, R., dos Santos, K., Jasencakova, Z., et al. (2001). Sequence organization of barley centromeres. Nucleic Acids Res. 29, 5029-5035. doi: 10.1093/nar/29.24.5029

Inaga, S., Naguro, T., Kameie, T., and Iino, A. (2000). Three-dimensional ultrastructure of in situ chromosomes and kinetochores of Tradescantia reflexa anther cells by scanning electron microscopy. 2. Whole mounted chromosomes and kinetochores of pollen moter cells and tapetal cells. Chromosome Sci. 4, 11-20.

Ishii, T., Karimi-Ashtiyani, R., Banaei-Moghaddam, A. M., Schubert, V., Fuchs, J., and Houben, A. (2015). The differential loading of two barley CENH3 variants into distinct centromeric substructures is cell type- and development-specific. Chromosome Res. 23, 277-284. doi: 10.1007/s10577-0159466-8

Jasencakova, Z., Meister, A., Walter, J., Turner, B. M., and Schubert, I. (2000). Histone H4 acetylation of euchromatin and heterochromatin is cell cycle dependent and correlated with replication rather than with transcription. Plant Cell 12, 2087-2100. doi: 10.1105/tpc.12.11.2087

Jiang, J. M., Birchler, J. A., Parrott, W. A., and Dawe, R. K. (2003). A molecular view of plant centromeres. Trends Plant Sci. 8, 570-575. doi: 10.1016/j.tplants.2003.10.011

Jovtchev, G., Borisova, B. E., Kuhlmann, M., Fuchs, J., Watanabe, K., Schubert, I., et al. (2011). Pairing of lacO tandem repeats in Arabidopsis thaliana nuclei requires the presence of hypermethylated, large arrays at two chromosomal positions, but does not depend on H3-lysine-9-dimethylation. Chromosoma 120, 609-619. doi: 10.1007/s00412-011-0335-8

Jovtchev, G., Watanabe, K., Pecinka, A., Rosin, F. M., Mette, M. F., Lam, E., et al. (2008). Size and number of tandem repeat arrays can determine somatic homologous pairing of transgene loci mediated by epigenetic modifications in Arabidopsis thaliana nuclei. Chromosoma 117, 267-276. doi: 10.1007/s00412007-0146-0

Kawabe, A., and Nasuda, S. (2005). Structure and genomic organization of centromeric repeats in Arabidopsis species. Mol. Genet. Genomics 272, 593-602. doi: 10.1007/s00438-004-1081-x

Koo, D. H., Sehgal, S. K., Friebe, B., and Gill, B. S. (2015). Structure and stability of telocentric chromosomes in wheat. PLOS ONE 10:e0137747. doi: 10.1371/journal.pone.0137747

Li, B. C., Choulet, F., Heng, Y. F., Hao, W. W., Paux, E., Liu, Z., et al. (2013). Wheat centromeric retrotransposons: the new ones take a major role in centromeric structure. Plant J. 73, 952-965. doi: 10.1111/tpj.12086

Liu, Z., Yue, W., Li, D. Y., Wang, R. R. C., Kong, X. Y., Lu, K., et al. (2008). Structure and dynamics of retrotransposons at wheat centromeres and pericentromeres. Chromosoma 117, 445-456. doi: 10.1007/s00412-008-0161-9

Ma, L., Vu, G. T., Schubert, V., Watanabe, K., Stein, N., Houben, A., et al. (2010). Synteny between Brachypodium distachyon and Hordeum vulgare as revealed by FISH. Chromosome Res. 18, 841-850. doi: 10.1007/s10577-010-9166-3

Marques, A., Ribeiro, T., Pavel Neumann, P., Macas, J., Novák, P., Schubert, V., et al. (2015). Holocentromeres in Rhynchospora are associated with genomewide centromere-specific repeat arrays interspersed among euchromatin. Proc. Natl. Acad. Sci. U.S.A. 112, 13633-13638. doi: 10.1073/pnas.1512255112

Martinez-Zapater, J., Estelle, A., and Sommerville, C. (1986). A highly repeated DNA sequence in Arabidopsis thaliana. Mol. Gen. Genet. 204, 4417-4423. doi: 10.1007/BF00331018

McNally, J. G., and Mazza, D. (2010). Fractal geometry in the nucleus. EMBO J. 29, 2-3. doi: 10.1038/emboj.2009.375

Metcalfe, C. J., Bulazel, K. V., Ferreri, G. C., Schroeder-Reiter, E., Wanner, G., Rem, W., et al. (2007). Genomic instability within centromeres of interspecific marsupial hybrids. Genetics 177, 2507-2517. doi: 10.1534/genetics.107.082313

Misteli, T. (2007). Beyond the sequence: cellular organization of genome function. Cell 128, 787-800. doi: 10.1016/j.cell.2007.01.028

Misteli, T. (2009). Self-organization in the genome. Proc. Natl. Acad. Sci. U.S.A. 106, 6885-6886. doi: 10.1073/pnas.0902010106

Neumann, P., Navratilova, A., Schroeder-Reiter, E., Koblizkova, A., Steinbauerova, V., Chocholova, E., et al. (2012). Stretching the rules: monocentric chromosomes with multiple centromere domains. PLoS Genet. 8:e1002777. doi: 10.1371/journal.pgen.1002777

Neumann, P., Schubert, V., Fuková, I., Manning, J. E., Houben, A., and Macas, J. (2016). Epigenetic histone marks of extended meta-polycentric centromeres of Lathyrus and Pisum chromosomes. Front. Plant Sci. 7:234. doi: 10.3389/fpls.2016.00234

Pecinka, A., Kato, N., Meister, A., Probst, A. V., Schubert, I., and Lam, E. (2005). Tandem repetitive transgenes and fluorescent chromatin tags alter local interphase chromosome arrangement in Arabidopsis thaliana. J. Cell Sci. 118, 3751-3758. doi: 10.1242/jcs.02498

Pecinka, A., Schubert, V., Meister, A., Kreth, G., Klatte, M., Lysak, M. A., et al. (2004). Chromosome territory arrangement and homologous pairing in nuclei of Arabidopsis thaliana are predominantly random except for NOR-bearing chromosomes. Chromosoma 113, 258-269. doi: 10.1007/s00412-004-0316-2

Plohl, M., Mestrovic, N., and Mravinac, B. (2014). Centromere identity from the DNA point of view. Chromosoma 123, 313-325. doi: 10.1007/s00412-0140462-0

Rabl, C. (1885). Über Zelltheilung. Morph. Jb. 10, 214-330.

Ribeiro, S. A., Vagnarelli, P., Dong, Y., Hori, T., McEwen, B. F., Fukagawa, T., et al. (2010). A super-resolution map of the vertebrate kinetochore. Proc. Natl. Acad. Sci. U.S.A. 107, 10484-10489. doi: 10.1073/pnas.1002325107

Sanei, M., Pickering, R., Kumke, K., Nasuda, S., and Houben, A. (2011). Loss of centromeric histone $\mathrm{H} 3$ (CENH3) from centromeres precedes uniparental chromosome elimination in interspecific barley hybrids. Proc. Natl. Acad. Sci. U.S.A. 108, 498-505. doi: 10.1073/pnas.1103190108

Schermelleh, L., Heintzmann, R., and Leonhardt, H. (2010). A guide to super-resolution fluorescence microscopy. J. Cell Biol. 190, 165-175. doi: $10.1083 /$ jcb. 201002018 
Schubert, I., Fransz, P. F., Fuchs, J., and de Jong, J. H. (2001). Chromosome painting in plants. Meth. Cell Sci. 23, 57-69. doi: 10.1023/A:1013137415093

Schubert, I., Shi, F., Fuchs, J., and Endo, T. R. (1998). An efficient screening for terminal deletions and translocations of barley chromosomes added to common wheat. Plant J. 14, 489-495. doi: 10.1046/j.1365-313X.1998. 00125.x

Schubert, V., Berr, A., and Meister, A. (2012). Interphase chromatin organisation in Arabidopsis nuclei: constraints versus randomness. Chromosoma 121, 369-387. doi: 10.1007/s00412-012-0367-8

Schubert, V., Klatte, M., Pecinka, A., Meister, A., Jasencakova, Z., and Schubert, I. (2006). Sister chromatids are often incompletely aligned in meristematic and endopolyploid interphase nuclei of Arabidopsis thaliana. Genetics 172, 467-475. doi: 10.1534/genetics.105.048363

Schubert, V., Lermontova, I., and Schubert, I. (2013). The Arabidopsis CAP-D proteins are required for correct chromatin organisation, growth and fertility. Chromosoma 122, 517-533. doi: 10.1007/s00412-013-0424-y

Screpanti, E., De Antoni, A., Alushin, G. M., Petrovic, A., Melis, T., Nogales, E., et al. (2011). Direct binding of Cenp-C to the Mis12 complex joins the inner and outer kinetochore. Curr. Biol. 21, 391-398. doi: 10.1016/j.cub.2010.12.039

Steedman, H. F. (1957). Polyester wax - new ribboning embedding medium for histology. Nature 179, 1345-1345. doi: 10.1038/1791345a0

Sullivan, B. A., and Karpen, G. H. (2004). Centromeric chromatin exhibits a histone modification pattern that is distinct from both euchromatin and heterochromatin. Nat. Struct. Mol. Biol. 11, 1076-1083. doi: 10.1038/nsmb845

Tůmová, P., Uzlikova, M., Wanner, G., and Nohynkova, E. (2015). Structural organization of very small chromosomes: study on a single-celled evolutionary distant eukaryote Giardia intestinalis. Chromosoma 124, 81-94. doi: 10.1007/s00412-014-0486-5

Wanner, G., Schröder-Reiter, E., Ma, W., Houben, A., and Schubert, V. (2015). The ultrastructure of mono- and holocentric plant centromeres: an immunological investigation by electron and structured illumination microscopy. Chromosoma 124, 503-517. doi: 10.1007/s00412-015-0521-1

Ward, P. (2002). "FISH probes and labelling techniques," in FISH, eds B. M. S. Beatty and J. Squire (Oxford: Oxford University), 5-28.

Watanabe, K., Pacher, M., Dukowic, S., Schubert, V., Puchta, H., and Schubert, I. (2009). The STRUCTURAL MAINTENANCE OF CHROMOSOMES 5/6 complex promotes sister chromatid alignment and homologous recombination after DNA damage in Arabidopsis thaliana. Plant Cell 21, 2688-2699. doi: $10.1105 /$ tpc.108.060525

Woodcock, C. L., and Ghosh, R. P. (2010). Chromatin higher-order structure and dynamics. Cold Spring Harb. Perspect. Biol. 2:a000596. doi: $10.1101 /$ cshperspect.a000596

Zhang, H., and Dawe, R. K. (2012). Total centromere size and genome size are strongly correlated in ten grass species. Chromosome Res. 20, 403-412. doi: 10.1007/s10577-012-9284-1

Conflict of Interest Statement: The authors declare that the research was conducted in the absence of any commercial or financial relationships that could be construed as a potential conflict of interest.

The reviewer ML and handling Editor declared their shared affiliation, and the handling Editor states that the process nevertheless met the standards of a fair and objective review.

Copyright (c) 2016 Schubert, Ruban and Houben. This is an open-access article distributed under the terms of the Creative Commons Attribution License (CC BY). The use, distribution or reproduction in other forums is permitted, provided the original author(s) or licensor are credited and that the original publication in this journal is cited, in accordance with accepted academic practice. No use, distribution or reproduction is permitted which does not comply with these terms. 